\author{
Classification \\ Physics Abstracts \\ $32.20 \mathrm{R}-34.50 \mathrm{H}-82.80$
}

\title{
An investigation of systematic errors in interactive EXELFS analysis
}

\author{
Mohammad Amirhamzeh Tafreshi, Christian Bohm and Stefan Csillag \\ Institute of Physics, University of Stockholm, Vanadisvägen 9, S-113 46 Stockholm, Sweden
}

(Received June 15, 1990; accepted October 08, 1990)

\begin{abstract}
Résumé. - L'analyse des modulations de structure présentes sur la partie haute énergie des seuils d'ionisation en spectroscopie des pertes d'énergie, les structures EXELFS, peut fournir des informations importantes, tant du point de vue structural que clinique, sur l'environnement atomique local. Cette analyse est cependant un peu complexe. Afin de déterminer les erreurs systématiques associées au choix des différents paramètres, nous avons évalué l'influence de quelques étapes sensibles au cours d'une analyse EXELFS interactive. Nous avons aussi essayé de définir quelques stratégies permettant de minimiser ces erreurs.
\end{abstract}

\begin{abstract}
Analyzing the fine structure modulations occurring on the high energy side of the ionization edges in atomic spectra, the EXELFS (EXtended Energy-Loss Fine Structure), can provide important structural and chemical information about the local atomic environment. The analysis procedure is, however, somewhat complicated. In order to determine systematic errors associated with different parameter choices, we have investigated some sensitive steps in interactive EXELFS analysis. We have also attempted to establish some strategies to minimize these errors.
\end{abstract}

\section{Introduction.}

The extended energy-loss fine structure occurs when inner shell electrons, emitted during external excitation, are reflected by the neighboring atoms so that emitted and reflected wave function components interfere. Whether the interference is constructive or destructive depends on the energy (or rather the wavelength) of the emitted electron, the distance to the neighbor atoms and the phase change introduced during the reflection. That is, if the electron energy and interatomic distances are such that the electron wave is reflected with an inverted phase after an integer number of periods, the interference will be constructive.

The interference will affect the excitation probability (the cross section) so that electrons experiencing constructive interference are promoted, while those experiencing destructive interference will be demoted. This behavior makes it possible to study the interference amplitude, as a function of electron energy, via the production cross section (Ref. [1]).

Approximating the ejected-electron wave function at the backscattering atom by a plane wave 
and assuming that multiple backscattering can be neglected, the interference amplitude can be described (Ref. [2]) by:

$$
X(k)=\sum_{j} \frac{N_{j}}{r_{j}^{2}}\left(\frac{f_{j}(k)}{k} \cdot \mathrm{e}^{-2 \cdot r_{\jmath} / \lambda_{\mathrm{i}}} \cdot \mathrm{e}^{-2 \sigma^{2} \cdot k^{2}}\right) \sin \left(2 k r_{j}+\phi_{j}(k)\right)
$$

where $N_{j}$ is the number of atoms in shell $j, r_{j}$ is the radius, $\phi_{j}(k)$ is the phase difference between the outgoing and reflected waves, $f_{j}(k)$ is the backscattering amplitude (which for light atoms can be approximated by $1 / k^{2}$, Ref. [3]), $\lambda_{\mathrm{i}}$ is the mean free path for inelastic scattering of the electron, and $\sigma_{j}$ is a disorder parameter.

The interference phenomenon has, depending on whether the excitation was due to incident photons (from synchrotron radiation) or electrons (from the electron gun in an electron microscope), given rise to two different spectroscopic methods, EXAFS and EXELFS, respectively. In this paper we are primarily concerned with EXELFS, although the EXAFS analysis is closely related.

In EXELFS approximately mono-energetic electrons penetrate a sample, during which some kinetic energy is lost due to inelastic processes. By recording the energy of the emerging electrons (energy-loss spectroscopy, Ref. [4]), it is possible to gain information about the atomic structure of the sample. The energy-loss spectrum can be separated into two parts, the low-loss region and the EXELFS region. Electrons in the low-loss region have lost kinetic energy to valence or plasmon excitations, while EXELFS electrons have lost energy to core electron excitations (as was described above). In the case of very thin samples (Fig. 2c), the former region mainly consists of unscattered electrons (zero-loss) that have not lost kinetic energy in the sample, i.e., the energy distribution is due to the energy distribution from the electron source.

For a thicker sample (Fig. 2a), the low-loss region will contain a wider energy distribution, including electrons that have lost encrgy to collective excitations of the valence electrons in the sample (e.g., plasmons).

With increasing sample thickness the probability for multiple scatterings also increases. These will cause multiple plasmon excitations and mixed plasmon-core excitations. In the latter case, core exciting electrons will also lose energy to plasmons, which will cause a shift in the associated EXELFS-spectrum. To a first order, plural scattering will affect the EXELFS spectrum in the same way as incident electrons with an energy distribution shaped like the low-loss distribution.

\section{EXELFS analysis.}

EXELFS spectra may be analyzed interactively or by statistical methods fitting predictions from assumed models to the data. In the latter method all corrections and transformations are included in the prediction. In the former method, however, these are performed one at a time under operator supervision. When analyzing an EXELFS spectrum from a given electron shell interactively, the following steps are recommended (Ref. [2]).

2.1 BACKGROUND SUBTRACTION. - Since the spectrum also contains contributions from other excited inner shell electrons, the first step is to remove those contributions. This is done by extrapolating and subtracting the energy distribution from electrons originating from lower energy shells. In an interactive analysis the extrapolation is obtained using a suitable function fitted to at least two arbitrarily chosen points on the distribution to be approximated (Ref. [5]). 
2.2 REMOVAl OF THE PLURAL SCATTERING FROM INNER-SHELl EDGES. - Plural scattering effects can drastically alter the observed shape of an inner-shell ionization edge, and their influence may have to be removed before the near-edge or the extended fine structure can be interpreted. Deconvolution techniques based on a Fourier transform will be used, since these are in many ways the most accurate and powerful methods (Ref. [6]). This step should actually have been done before the background subtraction but due to practical reasons it is more convenient to apply it afterwards. The errors introduced by this switch are minor.

2.3 CALIBRATION OF THE ENERGY-LOSS AXIS. - The next step is to compare an independently determined plasmon or inner-shell excitation energy with the distance, measured in channels, between the first (zero-loss) and the second peak (energy-loss due to plasmon or inner-shell excitation) to calibrate the energy-loss axis.

2.4 IDENTIFICATION OF THE EDGE POSITION. - In this step we examine the spectrum to identify the edge position (which has a well known energy). This is used as the origin of the energy-loss axis. A suitable portion of the EXELFS region, excluding near edge data points but otherwise chosen as large as possible, is then extracted for further analysis.

At this point the data reflects the energy distribution of the emitted electron wave, which may be assumed to be a smooth function of the encrgy, augmented by interference effects caused by the reflected wave. The smooth function corresponds to the intensity, which would have been measured in the absence of neighboring atoms (i.e., the free atom energy distribution).

2.5 ISOLATION OF THE OSCILLATORY COMPONENT. - In order to isolate the inteference amplitude from the production source of the interfering electrons, it is necessary to normalize the measured energy distribution, $J(E)$, with respect to an energy distribution corresponding to the free atom spectrum, $A(E)$, approximated by the smooth function we mentioned above.

$$
X_{1}(E)=\frac{J(E)}{A(E)}-1
$$

The result, which expresses the fractional enhancement (or reduction) of the electron emission due to interference effects, should then equal the interference amplitude.

2.6 SCALE CONVERSION. - The interference amplitude, initially presented as a function of energy, is now converted into a momentum representation. In this representation the interference amplitude can be decomposed into sinusoidal components corresponding to reflections from different neighboring atoms. This is described by the following relationship:

$$
X_{1}(K) \propto \sum_{j} \frac{N_{j}}{r_{j}^{2}} \sin \left(2 k r_{j}+\phi_{j}(k)\right)
$$

2.7 FOURIER TRANSFORMATION. - The interatomic distances are recovered from the interference data via a Fourier transform of the $X_{1}(k)$ using independently determined phase shift values (Ref. [3]). If these are not known a priori, the appropriate phase shifts will have to be estimated from suitable model compounds. 


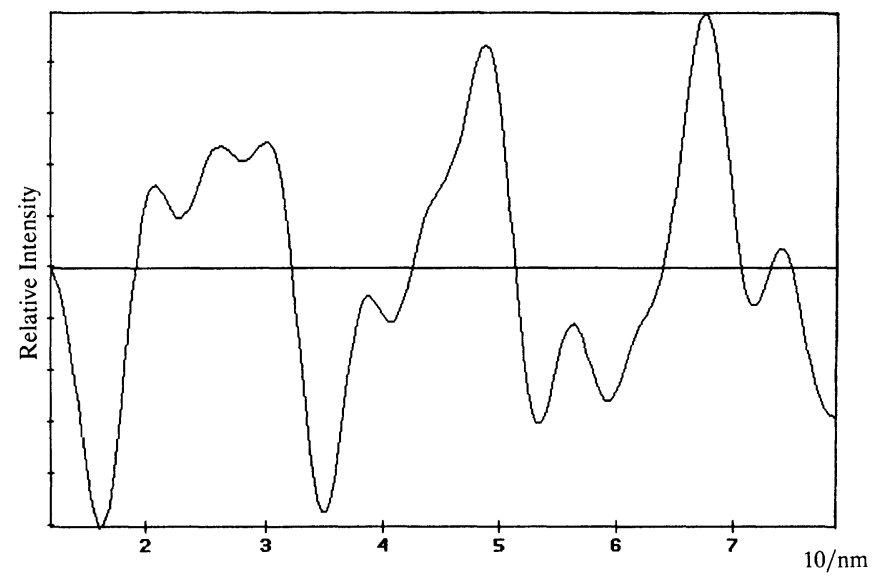

Fig. 1. - Simulated interference amplitude multiplied by $k^{3}$ corresponding to radial distances of $1.5,3.4$, and 5.2 Angstrom within an interval of 12 to $78 \mathrm{~nm}^{-1}$ (5.5 to $\left.232 \mathrm{eV}\right)$.

\section{Method.}

In this paper some systematic errors, introduced by using different assumptions in the analysis, are investigated by analyzing simulated data.

3.1 REMOVAL OF THE PLURAL SCATTERING FROM INNER-SHELL EDGES. - The low-loss region, which can be defined as the region corresponding to energy-losses below $50 \mathrm{eV}$ (Figs. 2a and 2c), mainly contains energy-losses caused by collective excitations of outer shells or free electrons (plasmon excitation). These excitations, which are particularly prevalent if thick samples are used, might also extract energy from the electrons causing the inner shell ionization (plural scattering). This leads effectively to a wider energy distribution of the incident electron, which may strongly affect the result of the EXELFS analysis. It is therefore desirable to study the effect of these excitations on the EXELFS spectrum.

In order to estimate this effect, we have used a simulated spectrum (Fig. 1) corresponding to a sample with neighbors at the radial distances 1.5, 3.4 and 5.2 Angstroms from the target atom, i.e. within an interval of 12 to $78 \mathrm{~nm}^{-1}(5.5$ to $232 \mathrm{eV})$. This spectrum was convoluted with three different low-loss spectra, a low-loss from a thick sample (Fig. 2a), a low-loss from a thin sample (Fig. 2c) and a Gaussian function (Fig. 2d). The results obtained are shown in figures $2 \mathrm{~b}, 2 \mathrm{~d}$, and 2f.

The results of Fourier transformation of the spectrum in figure $2 b$ gave the distances $0.3,1.4$, 3.6 and 5.2 Angstroms (compared with the original 1.5, 3.4 and 5.2 Angstroms). This deviation is not surprising, considering that the distortion caused by the convolution with the low-loss spectrum of figure $2 \mathrm{a}$, also can be regarded as a nonuniform amplification of the different frequency components emphasizing low frequencies.

Analysis of the "thin sample" spectrum in figure $2 \mathrm{~d}$ gave the distances 1.4, 3.4 and 5.1 Angstroms suggesting that in this case the deconvolution is not absolutely necessary.

A useful strategy when analyzing EXELFS data would be to examine the Fourier transform of the low-loss spectrum in the frequency interval of interest. If the transformed data indicates a uniform amplification in this region the deconvolution procedure can be avoided. 

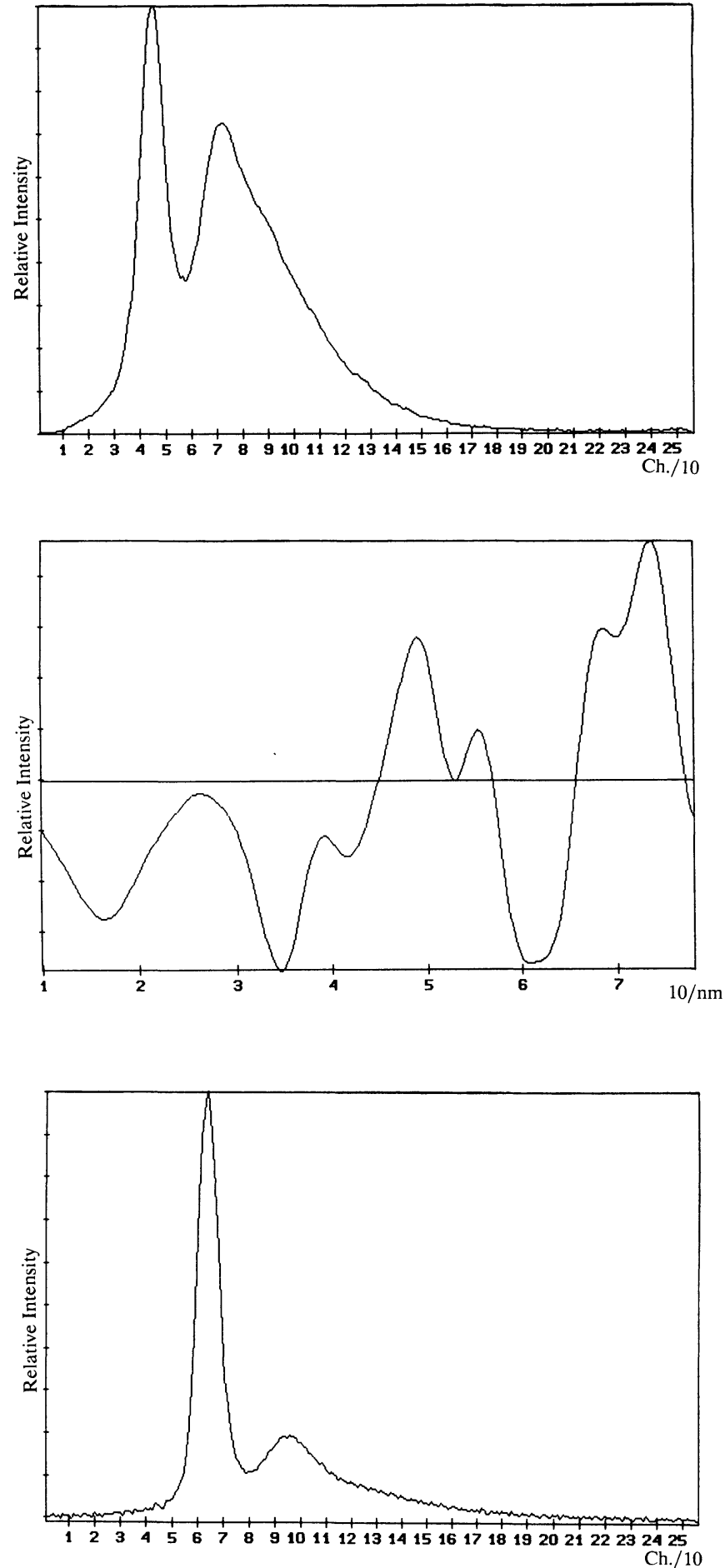

c)

Fig. 2. - Effect of convolution. (a), (c) and (e) are, the low-loss region of a thick sample, the low-loss region of a thin sample and Gaussian function, (b), (d) and (f) are convolution between (a), (c) and (e) with the simulated spectrum in figure 1. The energy per channel is $0.93 \mathrm{eV}$. 


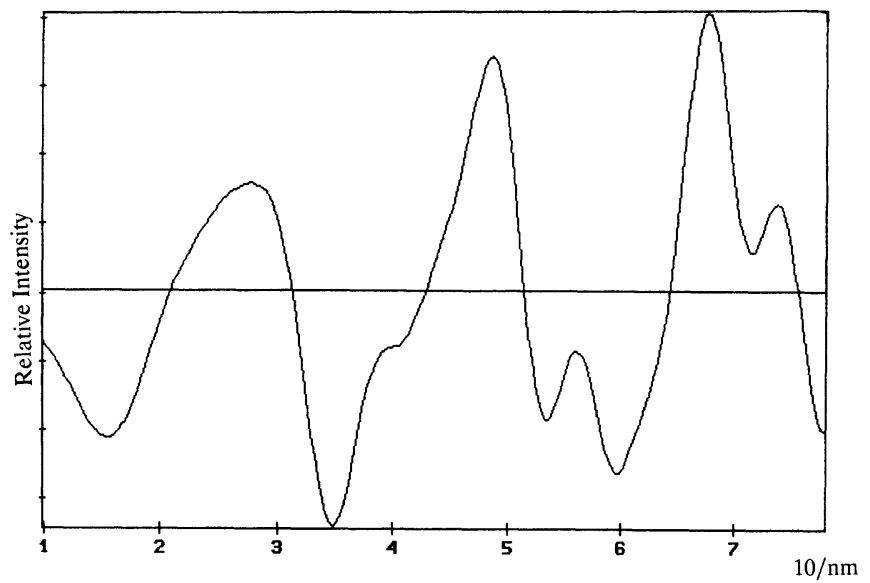

d)

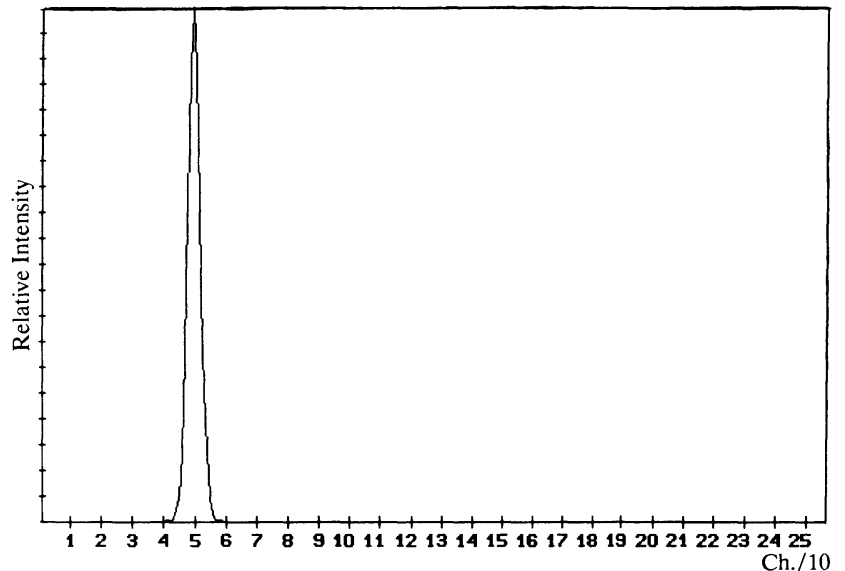

e)

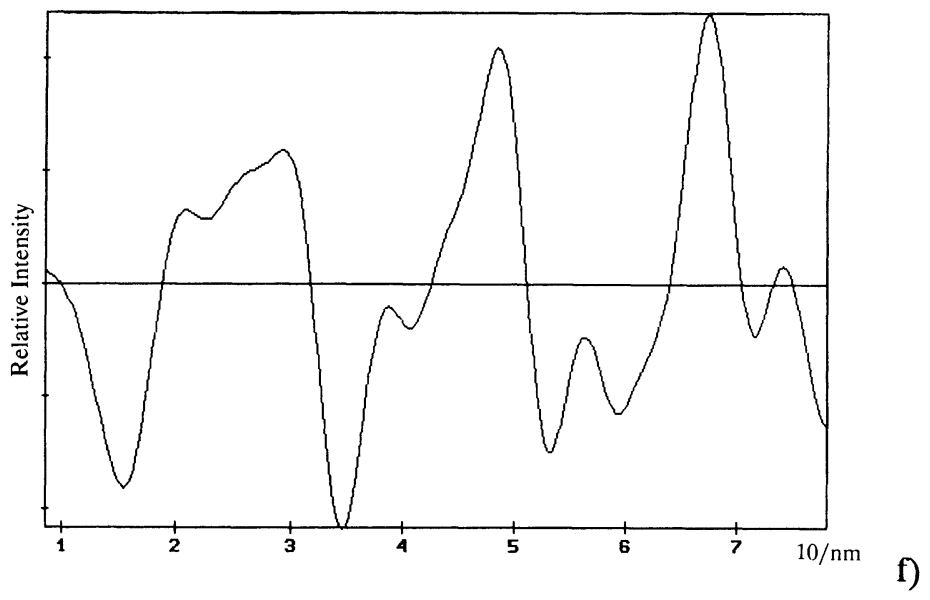

Fig. 2. - (continued) 
Figure 2f shows a convolution between the simulated spectrum and a thin Gaussian function. It can be seen that the result does not significantly differ from the originally simulated spectrum. Since the Fourier transform is a wide Gaussian which is roughly uniform over the region of interest. The analysis of this spectrum yields the undistorted distances 1.5, 3.4 and 5.2 Angstroms.

3.2 THE DECONVOLUTION PROCEDURE. - The deconvolution is carried out (according to the convolution theorem) by dividing the Fourier transform of the EXELFS spectrum by the Fourier transform of the low-loss spectrum. However, such "complete" deconvolution is in practice impossible because the high frequency noise components present in the Fourier transform of the EXELFS data will be amplified when divided by the small high frequency components of the Fourier transform of the low-loss spectrum. This may occur to the extent that the signal is completely obliterated.

One way to reduce the noise is to use the spectrometer resolution function as a filter i.e., deconvoluting the data with the low-loss spectrum and convoluting with the resolution function. This can sometimes lead to sufficient noise suppression but due to the extreme noise sensitivity of the deconvolution process it is often insufficient.

We can further reduce the noise by increasing the width of the filter function. However, the convolution with a wide filter function also degrades the resolution. It is therefore important to optimize the width of the filter function.

Another problem encountered during the analysis is caused by satellite peaks in the Fourier transform of the resolution function, which may introduce spurious high frequency components. This effect can be reduced if we approximate the resolution function with a Gaussian function with variable variance (Figs. 2 and 4). This filter function has the advantage of suppressing high frequency components.

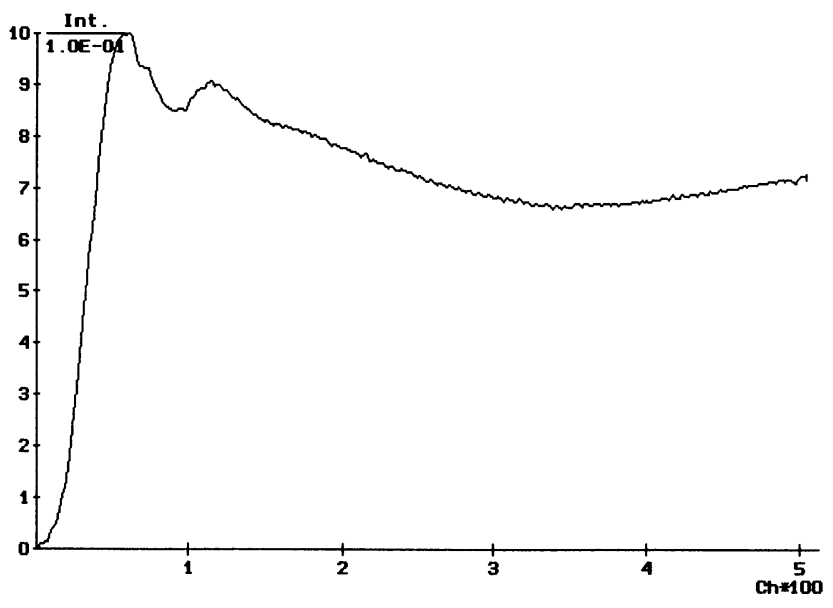

Fig. 3. - Energy-loss spectrum of a thin, amorphous carbon specimen after normalization and pre-edge background subtraction. The corresponding low-loss is in figure $2 \mathrm{c}$. 

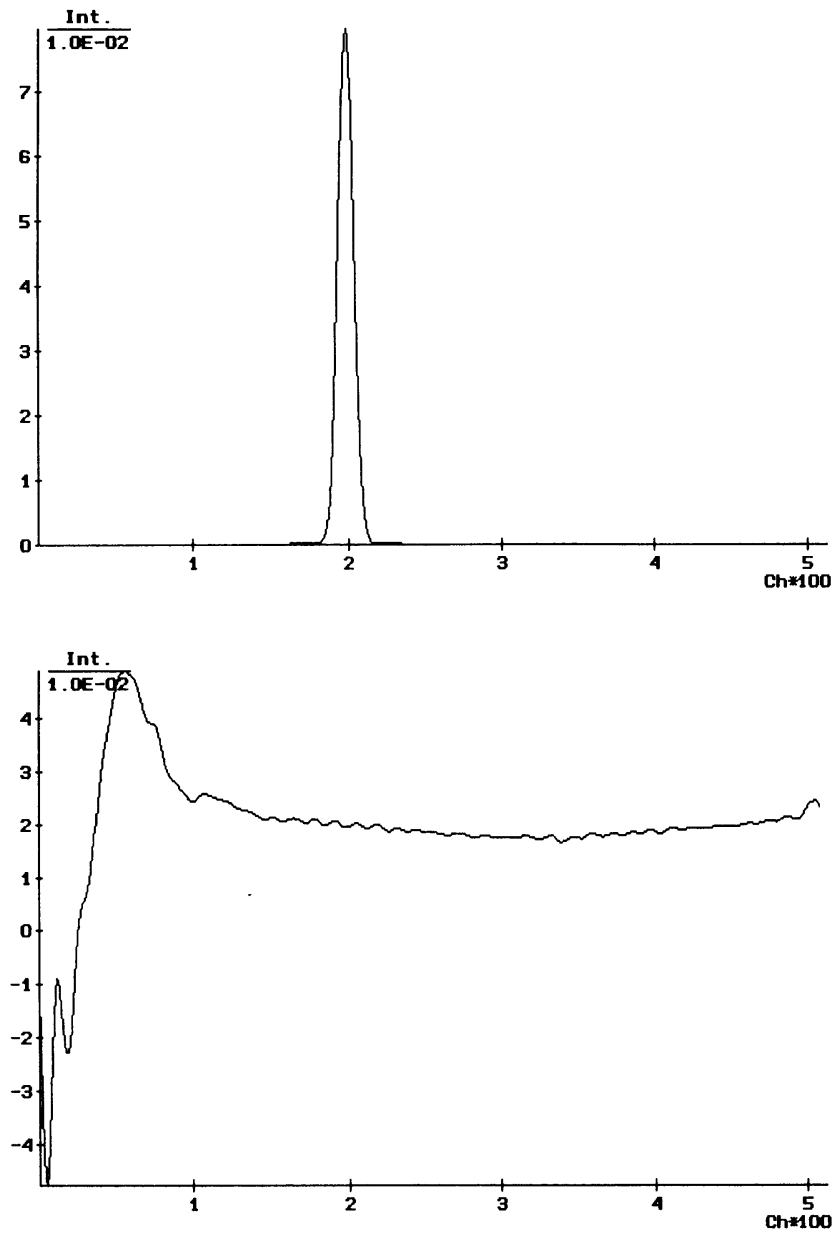

b)

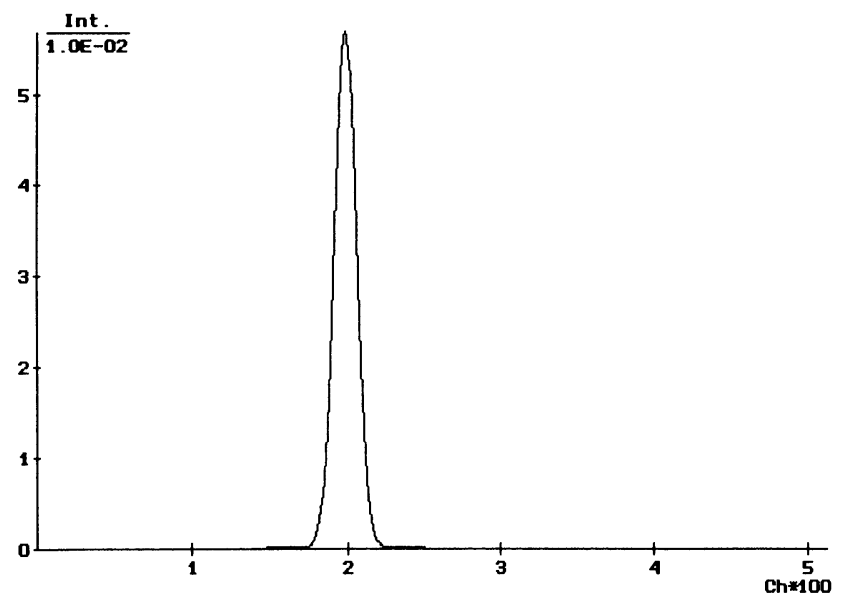

c)

Fig. 4. - The spectrum in figure 3, after deconvolution with low-loss spectrum and convolution with different width Gaussian functions. (b) The deconvoluted spectrum has been convoluted with the narrow Gaussian function in (a). (d) The deconvoluted spectrum has been convoluted with the broader Gaussian function in (c). 


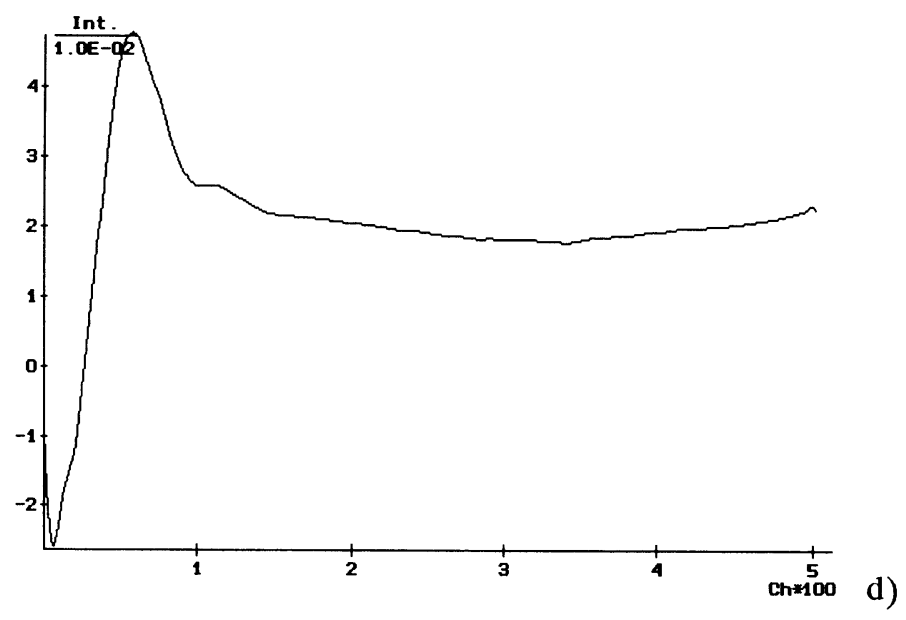

Fig. 4. - (continued)

\section{Calibration of the energy-loss axis.}

The calibration of the energy axis, which can be done by different methods, has direct effect on the result of the analysis. An uncertainty in the reference energy cause uncertainties in energy axis, $k$-axis and the distance axis. The relationship between the radial distance $(r)$ and the reference energy $\left(E_{\text {ref }}\right)$ allows us to estimate the resulting uncertainty in $r$.

$$
r \propto \frac{1}{\sqrt{E_{\mathrm{ref}}}}
$$

This indicates that for small deviations in $E_{\text {ref }}$

$$
\frac{\Delta r}{r}=-\frac{\Delta E_{\mathrm{ref}}}{2 \cdot E_{\mathrm{ref}}}
$$

For instance, when using the distance between zcro-loss and the plasmon peaks i.e. plasmon excitation energy, for calibration of the energy-loss axis, either the experimentally measured or the theoreticaly calculated plasmon energy should be chosen. Since, the experimental and theoretical results are not always in full agreement, an uncertainty is introduced.

As an example in the case of graphite the calculated plasmon excitation energy based on Jellium model is $22 \mathrm{eV}$, while the experimentally measured value is $27 \mathrm{eV}$. The assumption $E_{\mathrm{p}}=24.5 \pm 2.5 \mathrm{eV}$ then corresponds to $\pm 10 \%$ uncertainty in the energy-loss axis calibration, which in turn causes an uncertainty in distance determination of about $\pm 5 \%$.

\section{Identification of the edge position.}

The relation between edge position and wave number is

$$
k_{i} \propto \sqrt{i-p}
$$



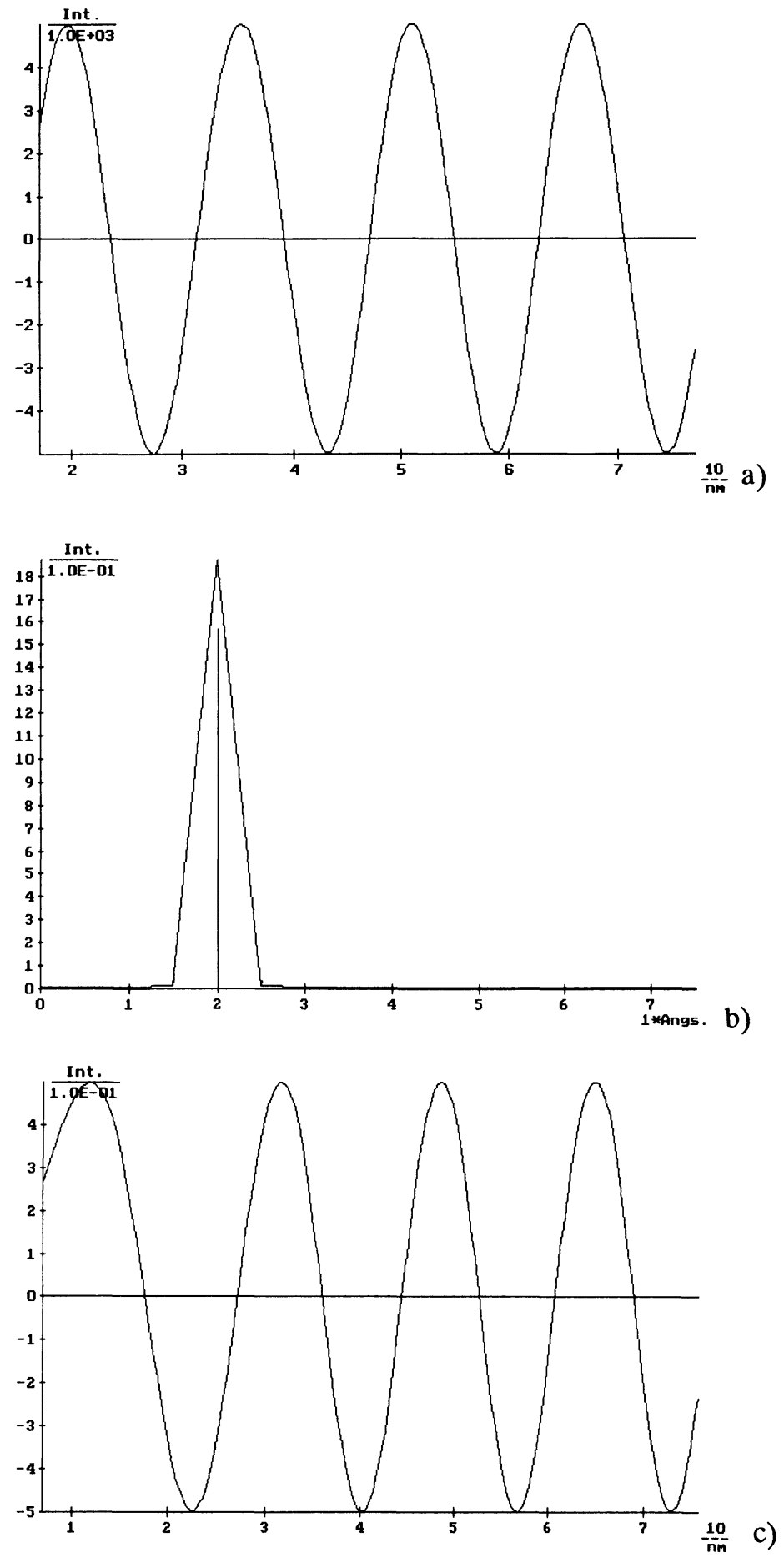

Fig. 5. - Effect of an inaccurate choice of the edge position. (a) A simulated EXELFS spectrum (with radial distances 2 Angstrom) after choosing the correct edge position, converting from energy to $k$-space, interpolating and multiplying by $k^{3}$. (c) Same as (a) but with the edge position chosen $9.3 \mathrm{eV}$ above the correct value. Observe the varying in period length, particularly in the beginning of the spectrum. (b) and (d) F.F.T. of the (a) respectively (c). 


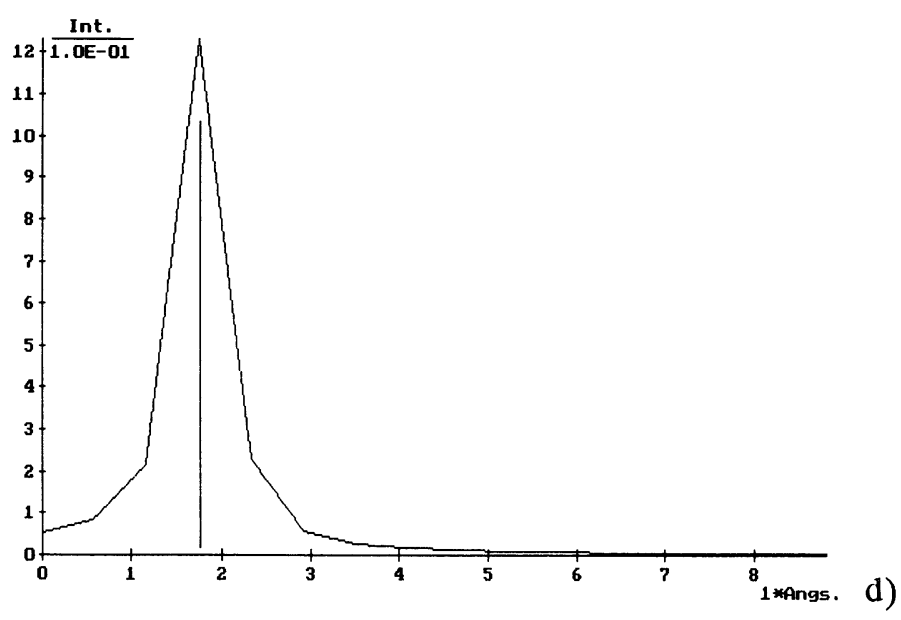

Fig. 5. - (continued)

where $i$ is the channel number, $k_{i}$ is the wave number of channel $i$ in $\mathrm{nm}^{-1}$ and $p$ is the channel number of the edge position.

For small deviations in $p$ the deviation in $k_{i}$ is

$$
\frac{\Delta k_{i}}{k_{i}}=-\frac{1}{2} \cdot \frac{\Delta p}{i-p}
$$

An inaccurate choice of the edge position $(p)$ causes a nonlinear deformation of the $k$-axis (Ref. [7]). This may be regarded as stretching and translation of the interval to adapt to the new $k_{\min }$ and $k_{\max }$ values followed by a non-linear deformation within the interval.

A closer examination of equation (6) reveals that it is more adequate to choose the edge position above the measured edge rather than below it; e.g., if we choose the edge position 10 channels below the real edge and $E_{\min } 30$ channels above the edge, then there is $-22 \%$ deviation in $k_{\min }$ value. Choosing instead the edge position 10 channels above the real position, the deviation in the $k_{\min }$ value will decrease to $13 \%$.

The effect of an inaccurate edge position decreases significantly with increasing distance from the edge. The proper determination of the real edge position (in the literature referred to as the $E_{0}$ problem) requires a fitting procedure to some known standard where $E_{0}$ is regarded as a free parameter or to some elaborate theoretical calculation (Ref. [8]). For the reasons mentioned above the errors can be minimized by choosing the analyzing interval as far above the edge as possible (Fig. 5c). This is, however, not always possible, since the EXELFS spectrum often extends only over a limited energy interval.

If a fast Fourier transform (FFT) algorithm is to be used, the $k$-sampling must be uniform, which means that some form of interpolation is needed. A nonlinear deformation of the $k$-axes caused by erroneous determination of the edge gives a deformed spectrum (compare Figs. 5a and 5c), which results in broadened peaks after FFT (compare Figs. 5b and 5d). This effect may possibly also be used as a criterion to find the real edge position. Even in this case the effect of the inaccurate choice of the edge position on the spectrum is reduced far from the edge position (Fig. 5c). 


\section{Fourier transfomation.}

Before computing the Fourier transform, values of $X(k)$ which lie outside a chosen range $\left(k_{\min }\right.$ to $\left.k_{\max }\right)$ are removed. The data is usually also weighted with $k, k^{2}$ or $k^{3}$ in order to compensate for the $k$-dependent amplitude decay of the modulations in the spectrum (see Eq. (1)), and windowed by a window function $(W)$ to minimize the truncation effects (Ref. [7]). A possible window function varies as $\cos ^{2} k$ at the ends of the data range but is flat over the central region.

The radial distribution function (RDF) of neighboring atoms is obtained by performing a Fourier transformation of the experimental EXELFS data. In practice a discrete Fourier transform is used, and the interatomic distances are deduced directly from the position of the peaks in the transformed spectrum. This discrete Fourier transform can be defined as follows

$$
X_{2}(r) \equiv \sum_{m=0}^{N-1} X_{1}\left(c \cdot m+k_{\min }\right) \cdot W(m) \cdot k^{p} \cdot \mathrm{e}^{2 i \pi m r / N} \quad p=1,2 \text { or } 3
$$

where $N$ is the number of the points to be transformed, which must be an integer power of 2, in order to be able to use the FFT algorithm; $c$ is a constant equal to $\left(k_{\max }-k_{\min }\right) /(N-1)$.

Since in most cases the interatomic distances obtained in the EXELFS analysis are in the range 1 to $3 \AA$ while the energy interval available for data analysis is between 150 and $400 \mathrm{eV}$ beyond the ionization edge (63 to $102 \mathrm{~nm}^{-1}$ ), the EXELFS modulation will mainly contain a small number of very low frequencies (Ref. [9]). The natural sampling $(\sim 1 / 2 \AA)$ is not sufficient to allow a determination of the main frequency components (Fig. $6 \mathrm{c}$ ). To provide a sufficient sampling density $(\sim 0.1 \AA)$ the energy interval must be extended to about $1.4 \mathrm{keV}$. The fact that we usually do not have any experimental data for energies over $400 \mathrm{eV}$ implies that the radial distribution function itself must be a low-pass filtered version of the "true" distribution. Another way of expressing this is to say that the estimated radial distribution function has a sinc $[(\sin x) / x]$ point spread function

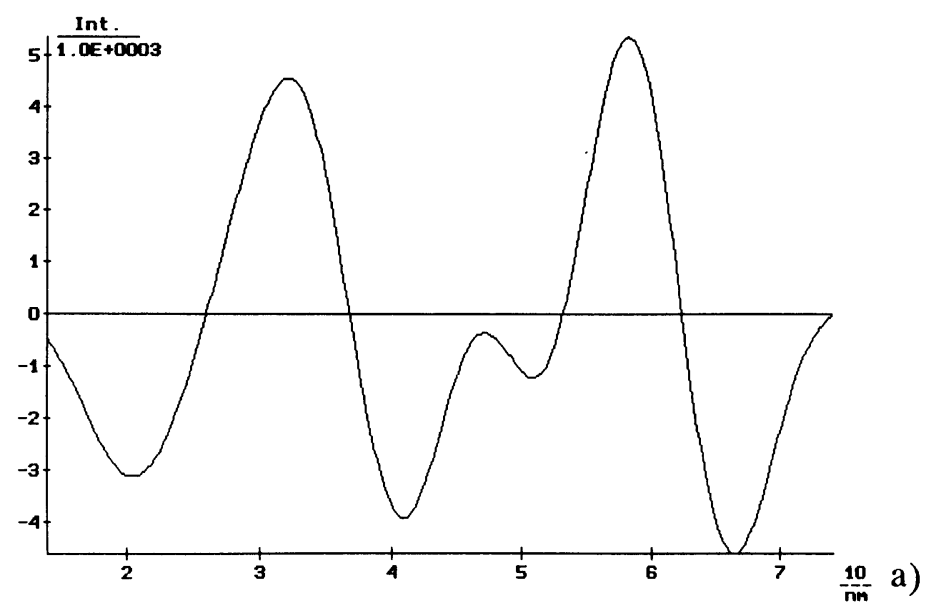

Fig. 6. - Effect of number of transformed points on the resolution. (6a) Simulated spectrum with radial distances at 1.25, 2.25 and2.75 Angstroms with 3, 6 and 3 neighboring atoms respectively. (6b) The figure in $6 \mathrm{a}$ multiplied by the window function. ( $6 \mathrm{c})$ and $(6 \mathrm{~d})$ are fast Fourier transform of the data in figure $6 \mathrm{~b}$, with 256 respective 16384 (zero-extended) data points. 

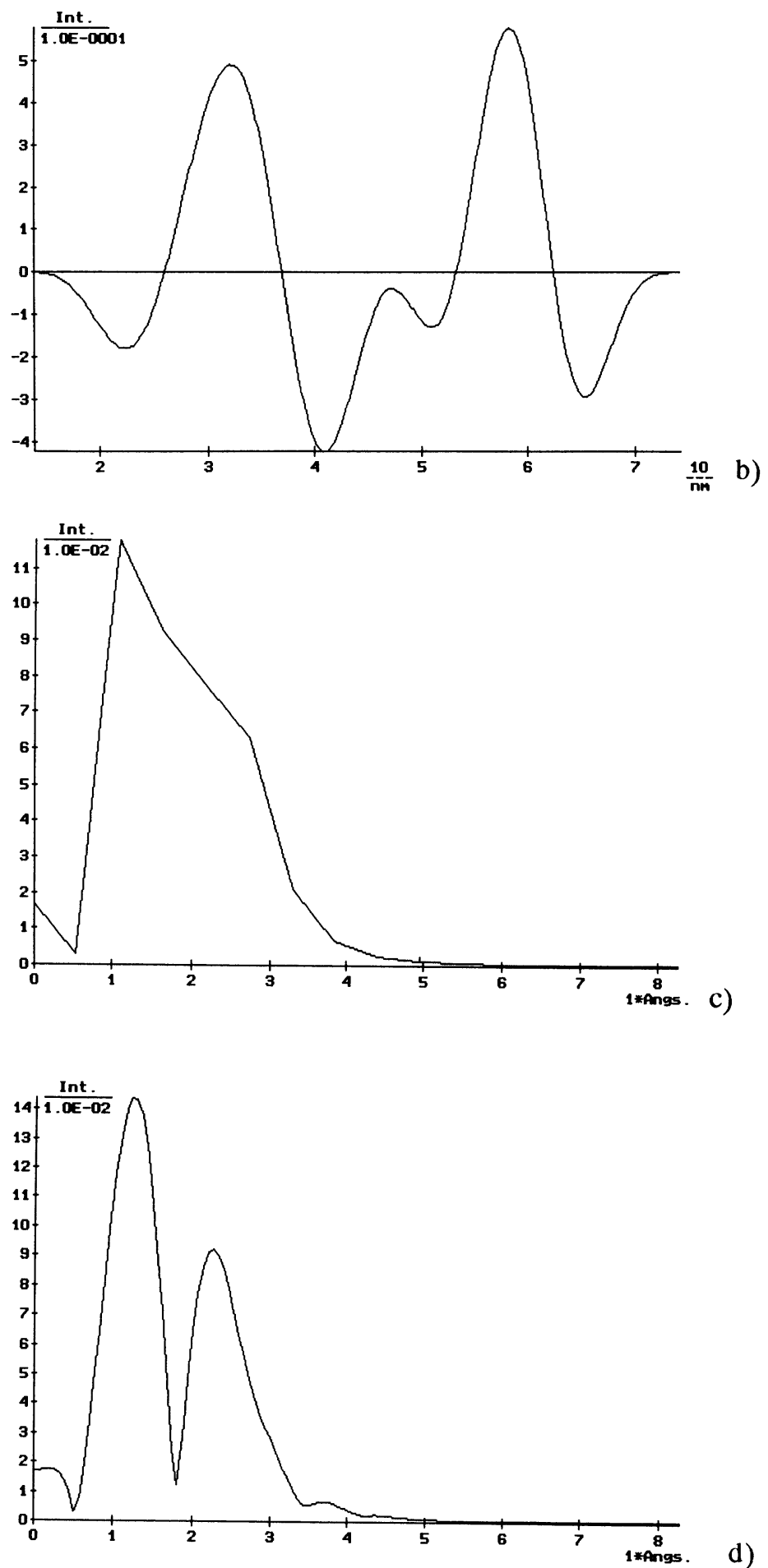

d)

Fig. 6. - (continued) 
(PSW). In practice, the high sampling density representation is obtained by zero-extending the energy axis before the Fourier transformation (Fig. 6d).

The PSW will also be affected by the weight function and the $k$ dependent amplitude in the expression for $X_{2}(k)$. Thus the sinc-PSW will be convoluted with the Fourier transform of the window function and a residual function, which corresponds to the part of the $k$ dependent amplitude that has not been compensated by $k^{p}$ correction (Eq. (8)).

The sinc component in the PSW will reduce the radial resolution due to the width of its central peak and cause interference problems when peaks are spaced with a distance so that a peak overlaps with its neighbors sinc-satellite peak (Ref. [10]). The sinc-satellites will also cause a number of smaller peaks in the background that must not be misinterpreted as atomic contributions.

The window function is used to reduce the PSW-satellites. A narrow window function will widen the PSW, but will also suppress satellites. The trade-off level depends on the spectrum at hand, which means that the optimum window function must be chosen from case to case.

\section{Concluding remarks.}

An initial deconvolution appears to be necessary in most cases in order to accurately analyze and interpret an EXELFS spectrum, especially when thick samples are used. The noise problem in the deconvolution process can be arbitrarily reduced by convoluting the deconvoluted data with a sufficiently broad Gaussian filter function. However this is done at the expense of degraded energy resolution.

A useful method for determining wheter deconvolution is necessary is to investigate the uniformity of the Fourier transformed low-loss data in the frequency region of interest. Nonuniform behavior advocates the use of deconvolution.

We also suggest that the method used in this work, analyzing simulated EXELFS-data, may be used to examine the neccesity to include the deconvolution procedure by analyzing and comparing simulated data from the experimentally predicted structure with and without deconvolution and compare the results. It is well known that the deconvolution procedure lack an unambiguous test criteria.

When considering the accuracy of the analysis it is important to bear in mind that an uncertainty in the calibration of the energy-loss axis causes an uncertainty in determination of the radial distances. Furthermore, to prevent undesirable effects caused by erroneous edge position determination, it is recommended that the analysis interval is chosen as far above the edge position as possible.

It also appears to be desirable to overestimate rather than underestimate the edge position. An inaccurate choice of the edge position results in deviations in the distance axis and in broadened peaks. The latter effect can also be used as a criterion (minimizing the peak width) to find the real edge position.

When interpreting the results of an analysis the resolution of the transformed spectrum is sometimes unsatisfactory. This is often due to the short data interval corresponding to the wavelengths of the principal modulations. By zero-extending the data outside the original data interval prior to the Fourier transform the apparent resolution will be improved. However, this improvement is achieved at the cost of increased transformation time and the appearance of smaller satellite peaks due to truncation effects. Special care must be taken when interpreting these peaks.

Interactive EXELFS analysis has proved reliable and fast in simple situations where the radial peaks are well resolved $(\sim 0.9 \AA$ for an interval of $220 \mathrm{eV})$. In complex and not well resolved situations, however, it has been found necessary to rely on more complicated statistical fitting procedures. 


\section{References}

[1] Colliex C., Cosslett V.E., Leapman R.D. and Trebbia P., Ultramicroscopy 1 (1976) 301.

[2] Csillag S., Ph.D., Thesis-Stockholm, 1980.

[3] TEO B.-K. and LeE P.A., J. Am. Chem. Soc. 101 (1979) 2815.

[4] CSILlag S., Johnson D.E. and STERN E.A., Extended Energy Loss Fine Structure studies in an electron microscope, In: EXAFS-spectroscopy, Eds. Teo and Joy, pp. 241-254.

[5] EGERTON R.F., Electron Energy-Loss Spectroscopy in the Electron Microscope, pp. 279-281.

[6] EgERTON R.F. and CROZIER P.A., Scanning Microsc. Suppl. 2 (1988) 245.

[7] LEE P.A. and BENI G., Phy. Rev. B15 (1977) 2862.

[8] BAXTER D.V., The Selection of $E_{0}$ for EXELFS Spectra of Disordered Systems. EXAFS and Near Edge Structure III, Ed. K.O., Hodgsen, B. Hedman, and J.E., Penner-Hahn (Springer-Verlag) pp. 77-79.

[9] Bourdillon A.J., El MAShri S.M. and ForTy A.J., Philos. Mag. 49 (1984) 341.

[10] Disko M.M., KrivaneK O.L. and ReZ P., Phys. Rev. B 25 (1982) 4252.

Cet article a été imprimé avec le Macro Package "Editions de Physique Avril 1990". 